\title{
IL-10 producing regulatory and helper T-cells in systemic lupus erythematosus
}

\author{
J. Geginat ${ }^{\mathrm{a}, *}$, M. Vasco ${ }^{\mathrm{a}}$, M. Gerosa ${ }^{\mathrm{b}, \mathrm{c}}$, S.W. Tas ${ }^{\mathrm{d}}$, M. Pagani ${ }^{\mathrm{a}, \mathrm{d}, \mathrm{e}}$, F. Grassi ${ }^{\mathrm{a}, \mathrm{f}}$, R.A. Flavell ${ }^{\mathrm{g}}$, \\ Pl. Meroni ${ }^{\text {h }}$, S. Abrignani ${ }^{\mathrm{a}, \mathrm{b}}$ \\ a INGM-National Institute of Molecular Genetics "Romeo ed Enrica Invernizzi”, Milan, Italy \\ ${ }^{\mathrm{b}}$ DISCCO, Department of Clinical Science and Community Health, University of Milan, Italy \\ ${ }^{\mathrm{c}}$ ASST Istituto G. Pini, Milan, Italy \\ ${ }^{\mathrm{d}}$ Amsterdam UMC, University of Amsterdam, Department of Rheumatology \& Clinical Immunology and Department of Experimental Immunology, Amsterdam Infection \& \\ Immunity Institute and Amsterdam Rheumatology \& immunology Center (ARC), Academic Medical Center, Amsterdam, the Netherlands \\ ${ }^{\mathrm{e}}$ Department of Medical Biotechnology and Translational Medicine, University of Milan, Italy \\ ${ }^{\mathrm{f}}$ Institute for Research in Biomedicine, Bellinzona, Switzerland \\ ${ }^{g}$ Department of Immunobiology, and Howard Hughes Medical Institute, School of Medicine, Yale University, New Haven, USA \\ ${ }^{\mathrm{h}}$ Istituto Auxologico Italiano, Milano, Italy
}

\section{A R T I C L E I N F O}

\section{Keywords:}

Systemic lupus erythematosus

IL-10

CD4 ${ }^{+}$T-cells

Autoantibodies

\begin{abstract}
A B S T R A C T
Systemic lupus erythematosus (SLE) is a highly heterogeneous autoimmune disease characterised by the production of pathogenic autoantibodies against nuclear self-antigens. The anti-inflammatory and tolerogenic cytokine Interleukin-10 appears to play a paradoxical pathogenic role in SLE and is therefore currently therapeutically targeted in clinical trials. It is generally assumed that the pathogenic effect of IL-10 in SLE is due to its growth and differentiation factor activity on autoreactive B-cells, but effects on other cells might also play a role. To date, a unique cellular source of pathogenic IL-10 in SLE has not been identified. In this review, we focus on the contribution of different $\mathrm{CD}^{+}{ }^{+} \mathrm{T}$-cell subsets to IL-10 and autoantibody production in SLE. In particular, we discuss that IL-10 produced by different subsets of adaptive regulatory T-cells, follicular helper T-cells and extra-follicular B-helper T-cells is likely to have different effects on autoreactive B-cell responses. A better understanding of the role of IL-10 in B-cell responses and lupus would allow to identify the most promising therapies for individual SLE patients in the future.
\end{abstract}

1. Systemic lupus erythematosus: a highly heterogeneous disease characterized by the production of autoantibodies to nuclear selfantigens

Systemic lupus erythematosus (SLE) is a prototypical multi-organ autoimmune disease, whose etiology is still unknown [1]. For the biology of IL-10 SLE is of particular interest, because it is generally assumed that IL-10 paradoxically plays a pathogenic role, and IL-10 neutralization may become a future therapeutic strategy [2]. Like many autoimmune diseases, SLE is a multi-factorial disease where both genetic and environmental factors contribute to the individual disease risk [1]. Genome-wide association studies have identified several risk loci, which have significantly contributed to our current understanding of lupus pathogenesis [3]. Among the identified genetic risk factors MHC/ HLA haplotypes show by far the strongest association, suggesting a key role for $\mathrm{CD} 4^{+} \mathrm{T}$-cells that recognise peptides presented on MHC class II complexes. Other genetic polymorphisms that suggest an involvement of T-cells and are shared with other autoimmune diseases are PTPN22, a tyrosine phosphatase inhibiting $\mathrm{T}$ cell receptor signalling, and STAT4, a transcription factor that promotes IFN- $\gamma$ production [4]. SLE is characterised by the breakdown of B cell tolerance and the production of autoantibodies, in particular against nuclear self-antigens. Although these antibodies can develop long before disease onset, they are believed to play a pathogenic role and are produced by autoreactive plasmablasts or plasma cells. They are mainly of the IgG class and are often somatically hyper-mutated, consistent with a role of CD4 help in their generation [5]. In addition, some polymorphisms in loci involved in B-cell activation, like BLK or BANK1, are also associated with SLE risk, suggesting also a role for intrinsic B cell hyper-activity. Consistent with a pathogenic role of B-cells in SLE, antibodies selectively targeting the $\mathrm{B}$ cell compartment (i.e. belimumab (anti-BAFF) and rituximab (anti-CD20)) provide therapeutic benefit [6-8] (Fig. 1).

\footnotetext{
* Corresponding author at: Fondazione Istituto Nazionale di Genetica Molecolare "Romeo ed Enrica Invernizzi" Via Sforza 3520122 Milano, Italy.

E-mail address: geginat@ingm.org (J. Geginat).
} 


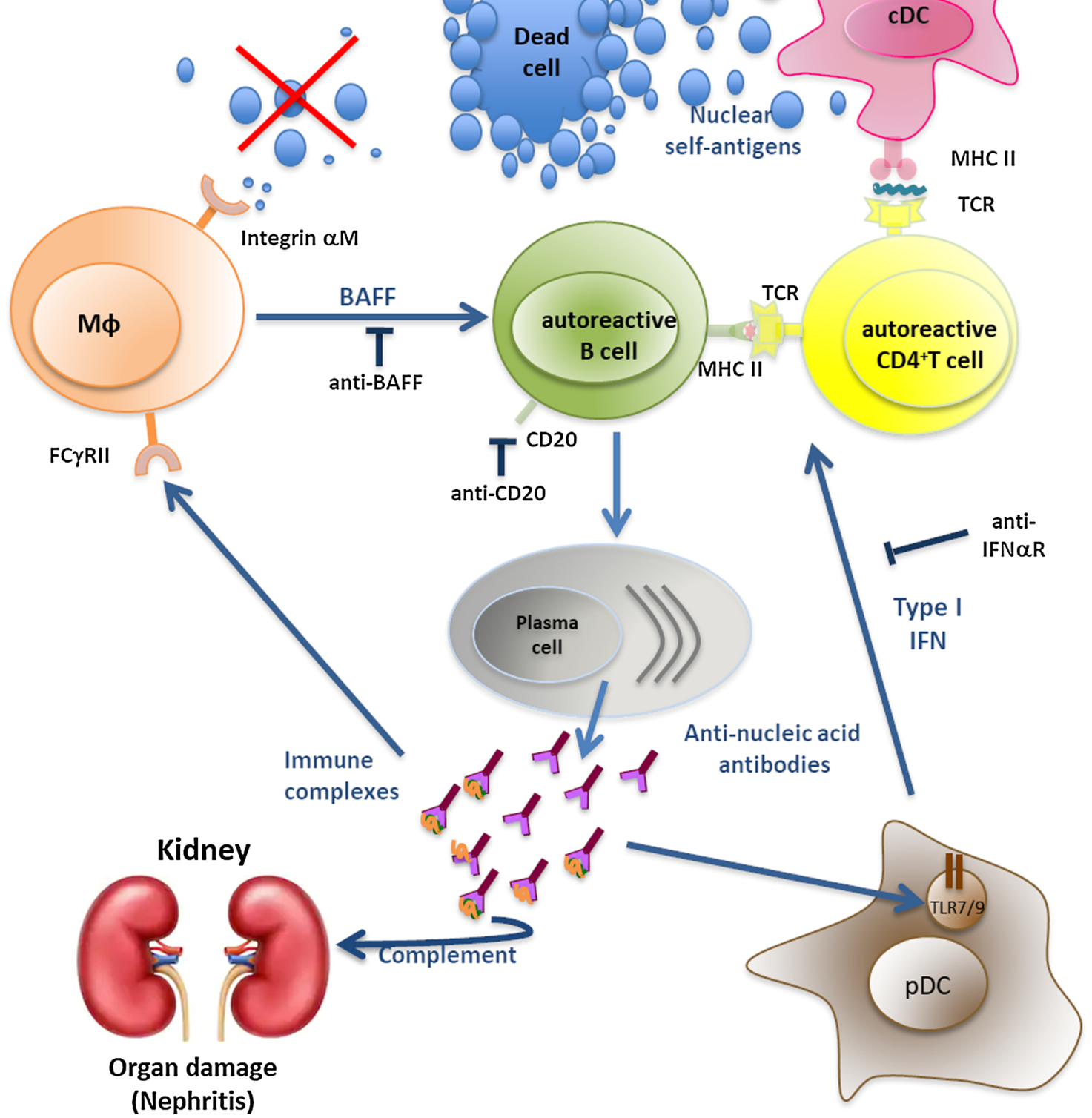

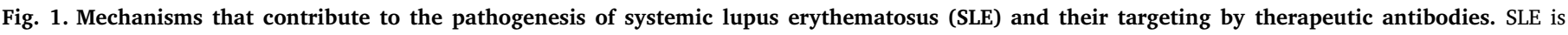

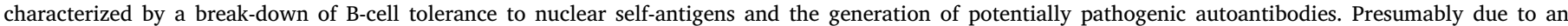

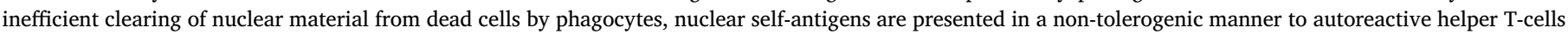

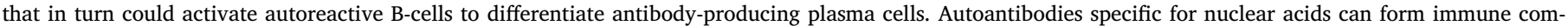

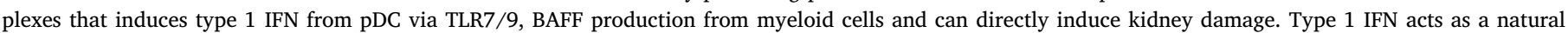

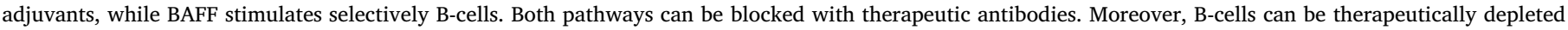
with anti-CD20 antibodies.

In addition to an increased activation of autoreactive B-cells by autoreactive T-cells, the genetic association studies also point to an important role of innate immunity in SLE [9]. In particular, the association of loci coding for Integrin $\alpha \mathrm{M}$ and $\mathrm{Fc} \gamma$ receptors with SLE suggests a role for inefficient phagocytosis and clearing of immune complexes. Notably, the association with Integrin $\alpha \mathrm{M}$ is apparently unique for SLE [4]. Also genetic deficiencies in the classical complement pathway, which are strongly associated with the risk of SLE, are thought to decrease the clearance of apoptotic cell debris and immune complexes, resulting in increased availability of nuclear self-antigens [9]. Finally, several loci coding for Interferon regulatory factors are associated with disease risk and SLE is characterised by an IFN signature in peripheral blood mononuclear cells. Immune complexes formed by autoantibodies and ribonucleoproteins activate plasmacytoid DC and induce type 1 IFN production, which acts in turn as a natural adjuvants to boost T- and B-cell responses [10]. Indeed, neutralization of type 1 interferon has therapeutic potential to treat SLE [11] (Fig. 1). In addition, immune complexes can deposit in the kidney and induce severe organ damage [12]. Taken together, the genetic risk factors are consistent with a scenario where inefficient clearing of material from dead cells leads to the activation of autoreactive T-cells, and consequently also of autoreactive B-cells that differentiate to autoreactive plasma blasts and secrete potentially pathogenic autoantibodies. These autoantibodies can form immune-complexes with their target antigens, 
and in the case of antibodies against nuclear antigens like doublestranded (ds) DNA induce a type 1 IFN response that further enhances the activation of autoreactive lymphocytes. In addition, cells of the innate immune system like macrophages can be activated by immune complexes and boost autoantibody production by secreting soluble Bcell stimulating factors like BAFF (Fig. 1).

While these mechanisms provide a plausible explanation for the systemic disease manifestations that may at least in part be mediated via autoantibody production and immune complex formation, it should be noted that SLE is a highly heterogeneous disease [13] that can affect different organs, like the skin, the joints, the CNS, the haematological compartment or the kidneys. Interestingly, the intestine is rarely affected in SLE, in spite of the fact that it is in general a highly sensitive organ to immune dysregulation. Nevertheless, the intestinal microbiome may play a critical role in SLE [14].

\section{The evidence for a pathogenic role of IL-10 in SLE}

The study of IL-10/IL-10R-deficient mice and humans has revealed that the non-redundant function of IL-10 signalling in humans and mice is to inhibit excessive inflammation in the gut in response to the intestinal microflora [2]. Also in organ-specific autoimmune diseases IL10 is thought to be protective [15], since it inhibits pathogenic inflammation and promotes self-tolerance. The notion that IL-10 plays a pathogenic role in SLE seems therefore paradoxical. However, besides its anti-inflammatory properties, IL-10 also acts as a growth and differentiation factor on cytotoxic lymphocytes [16] and B-cells [17], and promotes survival of autoreactive B-cells from germinal centres in vitro [18]. Notably, however, vaccination-induced IgG responses are normal in IL-10-deficient mice [19], suggesting that other STAT3-activating cytokines like IL-21 can substitute for IL-10 to induce differentiation of naïve B-cells to plasma cells, which has indeed been demonstrated in vitro [20-22].

Treatment of mice with anti-IL-10 antibodies provided insight into the role of IL-10 in autoreactive B-cell responses in vivo, as $\mathrm{CD}^{+}$ B1cells that can secrete T-independent, poly-specific and self-reactive "natural" IgM antibodies were killed upon anti-IL-10 treatment, while conventional B2-cells were spared [23]. However, it was later realised that the high affinity anti-dsDNA IgG autoantibodies that play a pathogenic role in SLE are produced predominantly by conventional B2cells [24]. Nevertheless, the same group also showed that treatment of lupus-prone NZB/W F1 mice with anti-IL-10 antibodies reduced serum levels of anti-dsDNA IgG autoantibodies and strongly increased survival, while administration of IL-10 accelerated autoimmunity [25]. The protective effect of IL-10 neutralization was mediated by increased TNF- $\alpha$ levels in this spontaneous model of lupus nephritis, but how increased TNF- $\alpha$ protected from disease was not addressed in this study. Of note, therapeutic TNF neutralization in patients can lead to autoantibody production and anti-TNF-induced lupus [26], suggesting that TNF plays a role in controlling autoreactive B-cell responses both in humans and mice. Interestingly, TNF can selectively kill auto-reactive $\mathrm{CD}^{+}{ }^{+} \mathrm{T}$-cells from various autoimmune patients, including those with lupus [27], but whether TNF could also kill autoreactive helper T-cells or B-cells from SLE patients remained unclear. Unfortunately, the role of IL-10 in murine lupus models is highly variable [28]. Thus, in the $\mathrm{MRL} / \mathrm{lpr}$ lupus model, where autoreactive lymphocytes expand due to impaired Fas-dependent killing, IL-10 is protective, since it inhibited pathogenic Th1 responses [29]. Patients with genetic defects in the Fas pathway develop however an autoimmune lymphoproliferative syndrome [30] which has largely distinct clinical features as compared to SLE. Nevertheless, given the huge heterogeneity of SLE [13], it is possible that the role of IL-10 in SLE patients also depends on clinical characteristics, like the involvement of different organs.

In SLE patients several lines of evidence point to a pathogenic role of IL-10. It is well documented that IL-10 serum levels are increased in SLE, at least in a proportion of patients [28]. Moreover, several groups found an association of IL-10 serum levels with disease activity scores $[28,31]$. Interestingly, a fraction of SLE patients produces autoantibodies against IL-10 [32], but if these anti-IL-10 antibodies were neutralizing was not investigated. IL-10 production is influenced by polymorphisms in regulatory elements of the IL-10 gene, and a large body of literature reports associations of single nucleotid polymorphisms (SNP) in the IL-10 gene and SLE risk [33-35]. Many independent studies found a significant association with SLE, although the odd's ratios were in general rather low, but this is not unusual for multifactorial diseases. Notably, the association with the most studied SNPs at position -1082/-819/-592 was not confirmed in all analysed patient cohorts [33]. A whole genome methylation analysis revealed that hypomethylation of the IL-10 promoter, which indicates increased accessibility for transcription (see Review by Kuchroo and colleagues in this special issue), was specifically associated with SLE, in particular in patients with higher disease activity [36]. In addition, immune cells from the peripheral blood of SLE patients spontaneously produce IL-10 [37], and the ratio of IL-10 to IFN- $\gamma$ production was reported to correlate with disease activity [38]. Intriguingly, B cells from SLE patients can spontaneously produce antibodies, and IL-10 was shown to be critical for this spontaneous antibody production in vitro, as well as for autoantibody production in humanised mice in vivo [39]. Moreover, anti-IL-10 treatment resulted in reduced human IgG levels in SCID mice implanted with hybridomas secreting anti-dsDNA antibodies [40]. These findings suggested that increased IL-10 production in SLE is not an epiphenomenon, reflecting merely a disease-associated hyperactivity of immune cells, but that IL-10 is part of a pathogenic mechanism that contributes to the disease. Consistently, Llorente et al. reported on 6 active SLE patients that were treated with a murine anti-IL-10 antibody [41]. Strikingly, although the patients, as expected, mounted an immune response to the mouse antibodies, they responded well to the treatment: joint and cutaneous involvement improved, corticosteroid dosage could be reduced and 5 patients had inactive disease after 6 months. Surprisingly, anti-dsDNA autoantibodies only decreased in one patient upon anti-IL-10 treatment [41]. Nevertheless, these results were sufficiently promising to motivate a randomised, multi-centred and placebo-controlled Phase II clinical trial with a higher number of SLE patients with a humanized monoclonal anti-IL-10 antibody (clinicaltrials.gov/ct2/show/NCT02554019). This antibody showed a favourable safety and tolerability profile, but the clinical efficacy has not been reported yet. The results of this trial will undoubtedly lead to a better understanding of the role of IL-10 in SLE.

\section{Are B cells the only relevant targets of IL-10 in SLE?}

Conditional IL-10R-deficient mice illustrated that the target cells of IL-10 in colitis were mainly intestinal macrophages [42], and potentially also Th17-cells [43]. Conversely, given the well-established pathogenic role of autoantibodies and immune complexes in SLE, it is generally assumed that IL-10R signalling in B cells and/or plasmablasts is key to its pathogenic effects. This notion is consistent with the capacity of IL-10 to induce survival, proliferation and differentiation of Bcells in vitro [44]. However, whether IL-10R signalling is required exclusively in the B-cell compartment in vivo has not been demonstrated yet. For T-cell dependent antibody production we observed that the role of IL-10R signalling in B-cells varied surprisingly according to the T-cell population that provided help [45]. In addition, it is still possible that also other cellular targets of IL-10 are important for SLE pathogenesis. Indeed, the rather surprising finding that anti-IL-10 antibody treatment in SLE rapidly reduced cutaneous manifestations in patients that maintained high levels of autoantibodies [41], suggests that IL-10 might play a pro-inflammatory role in tissues independently of its effects on B-cells. Notably, also CD8 ${ }^{+}$T-cells can be stimulated by IL-10 [46-48] (see review in this special issue by M. Oft), and they might play a pathogenic role in SLE as well [49-51]. Conversely, IL-10 has potent inhibitory effects on monocytes and dendritic cells (DC) [52], but this 
inhibitory function seems to be intact in SLE [53,54]. DC and macrophages could also be differentially affected by IL-10 [55,56], but if this interesting aspect is relevant for SLE is currently unknown.

IL-10 is involved in wound healing. IL-10R1 expression is predominantly expressed on immune cells [52], and this function could thus be mediated through the modulation of pro-inflammatory and fibrinogenic cytokines by immune cells. Nevertheless, several studies provided evidence for a direct effect of IL-10 on non-immune cells like fibroblasts, including IL-10R1 expression and IL-10-dependent signalling in these cells [57]. It should however be noted that a reported direct effect of IL-10 on keratinocytes [58], could not be confirmed by others [59]. Nevertheless, IL-10R1 expression was also detected on intestinal epithelial cells [60], consistent with the notion that IL-10 signalling is also relevant in non-immune cells. Studies with IL-10R reporter mice and cell-type specific knock-down of IL-10R signalling of IL-10-dependent lupus models may clarify this issue.

In summary, although the paradoxical notion that IL-10 plays a pathogenic role in SLE is increasingly recognised, the cellular targets remain to be defined, and the proposed mechanism, i.e. the induction of autoantibodies, might not explain all pathogenic effects in target tissues.

\section{Multiple cellular sources contribute to IL-10 production in SLE}

IL-10 is produced by most types of leukocytes [52] and in order to understand the mechanisms by which IL-10 promotes SLE it is thus important to identify the relevant cellular sources. In cell lineage-specific IL-10 k.o. mice, T-cells, but not macrophages or B-cells, were found to be the relevant source of IL-10 to prevent spontaneous colitis $[42,61]$. Conversely, a unique cellular source of pathogenic IL-10 in lupus has not been identified, also because, as discussed above, the role of IL-10 in murine lupus critically depends on the model that is employed. Studies on polymorphisms of the IL-10 locus that are associated with SLE in humans contributed to the identification of potentially involved signalling pathways, but did not point to a unique cellular source. Thus, the associations of SNPs in the IL-10 gene with IL-10 production was analysed by several groups, and many of them were shown to alter transcription factor binding to the IL-10 promoter [62]. IL-10 production varied according to SNPs at position 1082/-819/-592 upon LPS stimulation of total leukocytes and in EBV-transformed Bcells, indicating that these polymorphisms impact on IL-10 production by myeloid and B cells [62]. The GG genotype of the -1082 SNP was for instance shown to increase the binding of the Sp1 transcription factor and consequently of IL-10 production in LPS-stimulated B-cell lines [63]. Phagocytes produce IL-10 in response to dying cells [64], and in individuals with the SLE risk haplotype macrophages exposed to apoptotic cells displayed increased transcriptional activity of the IL-10 gene [65]. Another SNP $1 \mathrm{kB}$ downstream of IL-10 was shown to be associated with SLE risk in a very large patient cohort, and IL-10 production from this site was shown to be mediated by the transcription factor Elk-1. However, this pathway was again active in several different immune cell subsets, including monocytes, B-cells and T-cells [35]. Studies on the associations of polymorphisms with IL-10 levels produced by polyclonal T-cells [66] are complicated by the fact that the individual antigen exposure is expected to have a strong impact on Tcell IL-10 production [67].

The analysis of IL-10 production by different leukocyte subsets did also not lead to the identification of a unique cellular source of IL-10. The spontaneous IL-10 production of peripheral blood mononuclear cells from SLE patients was shown to be mainly due to monocytes and B-cells [37], with a variable contribution of T-cells [68]. Notably, immune complexes induce not only production of pathogenic type 1 IFN from pDC [11], but also IL-10 from monocytes/macrophages via FCR $\gamma \mathrm{II}$ $[69,70]$. Therefore, it seems likely that this pathway contributes significantly to the increased serum levels of IL-10, in particular in patients with high disease activity. Moreover, IFN- $\alpha$ in serum from SLE patients instructed monocytes to promote B-cell IgG production, and this B helper effect was dependent on IL-10 and BAFF [71]. IL-10 is also produced by DC, and IL-10 production by DC was found to be dysregulated in murine lupus caused by enhanced IRF4 activity [72]. In the human DC compartment, IL-10 is selectively produced by $\mathrm{CD} 1 \mathrm{c}^{+} \mathrm{DC}$ (cDC2) and has a unique regulation [48], but if cDC2 have altered IL-10 production in SLE has not yet been investigated. Finally, IL-10 production by B-cells might promote plasma blast differentiation in an autocrine feed-forward loop [73]. However, IL-10 in the B-cell compartment is also produced by regulatory B-cells (see review by Fillatreau and colleagues in this special issue) that potentially have a protective role, but are functionally impaired in SLE [74-76].

In summary, the current evidence suggests that multiple cellular sources contribute to the excessive IL-10 production in lupus, including myeloid cells, B-cells and also T-cells. We will focus on the role of different $\mathrm{CD} 4{ }^{+} \mathrm{T}$-cell subsets for the remainder of this review.

\section{IL-10 producing regulatory and helper T-cell subsets}

In the $\mathrm{T}$-cell compartment, $\alpha / \beta \mathrm{TCR}^{+} \mathrm{CD} 4{ }^{+} \mathrm{T}$-cells produce the highest levels of IL-10. In the $\mathrm{CD}^{+}{ }^{+} \mathrm{T}$-cell compartment, IL-10 is produced at particular high levels by regulatory T-cells, which in most but not all cases inhibit or skew B-cell responses [77-81]. IL-10 is however also produced by non-regulatory T-cells which might promote inflammation (see Review by Huber\&Gagliani and colleagues in this special issue), in particular in the spleen [82]. T helper cells are highly abundant and some possess B helper capabilities [83-87]. All human T helper subsets can produce IL-10 [88-90] and could thus make an important contribution to total IL-10 production, in particular upon cognate interaction with auto-reactive B-cells. Interestingly, besides $\mathrm{CD} 4{ }^{+} \mathrm{T}$-cells also $\mathrm{CD}^{-}{ }^{-} \mathrm{CD} 8^{-} \alpha / \beta \mathrm{TCR}^{+} \mathrm{T}$-cells have been shown to spontaneously induce anti-dsDNA autoantibody production from autologous B-cells in SLE patients [91]. However, if IL-10 was produced by these unconventional autoreactive T-cells and if IL-10 promoted autoantibody generation was not investigated.

\subsection{IL-10 producing regulatory $T$-cells suppress $B$-cell responses, but are dysfunctional in SLE}

The best-defined regulatory $\mathrm{T}$-cells express the lineage-defining transcription factor FOXP3 [92], and FOXP3 ${ }^{+}$Tregs can produce high levels of IL-10 in humans and mice, in particular in the mucosa and in the skin [79,93-96]. FOXP3 is quite selectively expressed in Tregs, and genetic deficiencies in its gene causes multi-organ autoimmune manifestations and autoantibody production both in humans and mice, indicating that $\mathrm{FOXP}^{+}$Tregs are required to maintain self-tolerance and to suppress autoreactive B cells $[97,98]$. Consistently, FOXP3 ${ }^{+}$Tregs suppress B-cell responses, and specialised B suppressor Tregs (Tfr) are present in germinal centres of secondary lymphoid organs [99]. Therefore, a defect in FOXP ${ }^{+}$Tregs was expected to contribute to SLE pathogenesis, but contradictory results were published on the numbers and functionalities of Tregs in SLE [100]. Notably, patients that have only a partial defect in FOXP3 expression also develop anti-enterocyte autoantibodies and non-IPEX autoimmune enteropathy [101,102]. These findings indicate that FOXP $3^{+}$Tregs are required to prevent autoimmunity in the small intestine, and that a defect in FOXP $3^{+}$Tregs is insufficient to induce lupus-like symptoms. It is thus possible that regulatory T-cells that do not express FOXP3, but also suppress B-cell responses, play an important role in lupus. Indeed, IL-10 is not only produced by FOXP ${ }^{+}$Tregs, but is the defining cytokine of the so-called "type 1 regulatory" (Tr1) T-cells [103]. Cells that satisfy the broad current definition of $\mathrm{Tr} 1$-cells, i.e. $\mathrm{CD} 4{ }^{+} \mathrm{T}$-cells with $\mathrm{IL}-10$ producing capacities and suppressive functions that are distinct from established T-cell subsets [104], can be generated in humans with different protocols in vitro. Unexpectedly, Tr1-cells that were generated upon TCR activation and co-stimulation via the complement receptor CD46 were 
shown to promote B-cell responses [77]. How in vitro generated $\operatorname{Tr} 1$ cells are related to in vivo occurring Tr1-cells is however difficult to address, in particular in complex human diseases like SLE [105]. In inflamed human tonsils, the most accessible secondary lymphoid organ to study human B-cell responses, Tr1-cells that produce high amounts of IL-10 ex vivo could be identified [79,106]. Importantly, tonsillar Tr1cells and Tregs inhibited B-cell responses, but this suppression was independent of IL-10 production. Similar findings were reported independently for tonsillar Tr1-cells identified according to expression of either Lag3 or CCR5/PD1 $[79,106]$. This is not just a trivial confirmation, since $\mathrm{CCR} 5^{+} \mathrm{PD} 1{ }^{+}$and $\mathrm{Lag} 3^{+}$Tr1-cells differ in the expression levels of surface Lag3 and IL-7R, as well as the putative lineage-defining transcription factor Eomes [107], and there is consequently little overlap between these two Tr1-cell population in human blood [93,108]. Importantly, in marked contrast to $\mathrm{CD} 4{ }^{+} \mathrm{IL}-7 \mathrm{R}^{+} \mathrm{T}$-cells, both Foxp $3^{+}$Tregs and Eomes ${ }^{+}$Tr1-cells are largely unable to up-regulate CD40 L expression $[79,108,109]$. This defect has major implications for the regulation of B-cell responses, since humans and mice with genetic defects in the CD40/CD40 L pathway have impaired IgG induction $[110,111]$. Conversely, the absence of the most potent B helper cytokine IL-21 results only in a partial reduction of IgG production [112]. Interestingly, provision of exogenous $\mathrm{CD} 40 \mathrm{~L}$ was sufficient to induce Bcell IgG production in the presence of Tr1-cells, but not of FOXP $3^{+}$Tregs [79]. Thus, the lack of CD40 L in Eomes ${ }^{+}$Tr1-cells is mandatory for their capabilities to suppress B-cell responses. Moreover, the presence of CD40 L is sufficient to convey B helper functions to Tr1cells, presumably because -unlike FOXP ${ }^{+}$Tregs- Tr1-cells secrete high levels of IL-21. In SLE patients, Eomes ${ }^{+}$Tr1-cells were found to be increased but dysfunctional, since they were unable to suppress B-cell IgG production [79]. Conversely, Lag $3^{+}$Tr1-cells are controlled by the transcription factor Egr-2 [113, 114], suppressed B-cell responses via cell-cell contact and TGF- $\beta 3$, and were reduced in SLE patients $[80,115]$. Eomes ${ }^{+}$Tr1-cells even provided B-cell help in some patients, most likely due to residual $\mathrm{CD} 40 \mathrm{~L}$ expression. In contrast, FOXP $3^{+}$Tregs from the same patients suppressed IgG production efficiently, indicating that the defect was Tr1-specific [79]. Notably, Th1 effector memory cells contain a fraction of Eomes ${ }^{+} \mathrm{T}$-cells that share some key characteristics with Eomes ${ }^{+} \operatorname{Tr} 1$-cells, but unlike $\operatorname{Tr} 1$-cells they express IL-7R and CD40 L, and they produce only low levels of IL10 [108]. These Eomes ${ }^{+}$Th1-cells may represent pre-cursors of Eomes ${ }^{+} \operatorname{Tr} 1$-cells, and based on this hypothesis it seems plausible that Eomes ${ }^{+}$Th1-cells fail to efficiently down-regulate CD40 L upon differentiation to $\operatorname{Tr} 1$ effector cells in SLE patients. Since IL-27 down-regulated CD40 L in synergy with Eomes [108], it seems plausible that IL27-dependent Tr1-cell generation is disturbed in SLE. Indeed, a role for IL-27 in lupus has been proposed [116-118]. Alternatively, the defect might be related to dysfunctional Bregs that fail to induce Tr1-cells in SLE [75,119], but whether Breg-induced Tr1-cells express Eomes and/ or require IL-27 remains to be addressed.

Overall, these studies suggest that dysfunctional IL-10 producing regulatory B and T lymphocytes are key to SLE pathogenesis (Fig. 2).

\subsection{Follicular helper T-cells are activated in lupus and contribute to autoantibody production}

As stated above, IL-10 is also produced helper T-cells, which may thus contribute to systemic IL-10 accumulation in SLE patients. It should be noted that IL-10 has a peculiar regulation as compared to other cytokines, since resting helper T-cells produce IL-10 preferentially at late time points $[79,120]$. Consequently, brief polyclonal standard stimulation is well suited to induce IL-10 production in activated and regulatory T-cells, but not in quiescent helper T-cells that represent the large majority of T-cells in human peripheral blood. A plausible teleological explanation for the different kinetics of IL-10 production by regulatory and helper T-cells is that rapid IL-10 production is required to inhibit DC, macrophages and potentially also Th17 effector cells that express high levels of IL-10R [43,121,122]. Conversely, late IL-10 production is tailored to promote B-cell and CTL responses, since naive B-cells and $\mathrm{CD}^{+}{ }^{+} \mathrm{T}$-cells first have to up-regulate IL-10R expression upon activation. IL-10 was originally found to be produced by Th2-cells [123], which could thus induce B-cell responses via IL-10 and IL-4. Indeed, a shift to Th2-cells in SLE patients was originally reported [124]. After the discovery of Th17-cells and of $\mathrm{T}_{\mathrm{FH}^{-} \text {-cells the original }}$ conceptual Th1/Th2 framework has however been extensively revised. It is now well established that $\mathrm{T}_{\mathrm{FH}}$-cells are the professional $\mathrm{B}$ helper effector T-cells and reside in germinal centres of secondary lymphoid organs. They co-express CXCR5, ICOS, BCL6 and produce high levels of IL-21 [125]. Interestingly, human $\mathrm{T}_{\mathrm{FH}}$-cells produce some IL-4 [126], and in immune responses against parasites murine $\mathrm{T}_{\mathrm{FH}}$-cells are derived from Th2-cells [127]. Given their potent $\mathrm{B}$ helper functions, $\mathrm{T}_{\mathrm{FH}}$ were obvious candidates for the induction of pathogenic autoantibodies in SLE [128]. Indeed, in the sanroque lupus mouse model $\mathrm{T}_{\mathrm{FH}}$-cells are required to induce anti-dsDNA autoantibodies [129]. Since it is difficult to obtain lymphoid organ tissue samples of SLE patients, very little is known about canonical $\mathrm{T}_{\mathrm{FH}^{-}}$-cells in SLE. However, circulating $\mathrm{CXCR}^{+}{ }^{+} \mathrm{T}$-cells possess potent $\mathrm{B}$ helper capabilities and were proposed to be derived from $\mathrm{T}_{\mathrm{FH}}$ effector cells $[86,130]$. Notably, however, circulating CXCR5 $^{+}$T-cells are pre-dominantly non-polarised central memory T-cells [131] and express only low levels of ICOS, IL-21 and BCL6. A fraction of circulating CXCR5 ${ }^{+}$T-cells express however PD1 and some BCL6, and thus appear to be more closely related to $\mathrm{T}_{\mathrm{FH}}$-cells that are found in germinal centres $[130,132]$. Indeed, in human SLE, $\mathrm{CXCR}^{+} \mathrm{T}_{\mathrm{FH}}$ cells display a more activated $\mathrm{PD} 1^{+} \mathrm{CCR} 7^{10} \mathrm{~T}_{\mathrm{FH}}$-like phenotype, and expansions of different $\mathrm{CXCR} 5^{+} \mathrm{T}$-cell populations was reported in SLE patients [133-136]. The capacity of $\mathrm{T}_{\mathrm{FH}^{-}}$-cells to induce autoantibodies in SLE was however assessed in the presence of exogenous TCR agonists like anti-CD3/28 antibodies, and whether circulating CXCR5 ${ }^{+} \mathrm{T}_{\mathrm{FH}^{-}}$-like cells are autoreactive and can productively interact with autoreactive B-cells, a key point to understand their role in SLE, is still unclear. In contrast to previous reports we observed overall reduced frequencies of CXCR5 ${ }^{+} \mathrm{T}$-cells in SLE patients [45], but purified $\mathrm{CXCR}^{+} \mathrm{T}_{\mathrm{FH}}$-like cells from SLE patients were able to spontaneously induce IgG from autologous B-cells, suggesting that they may contribute to autoantibody production. IL-10 produced by $\mathrm{T}_{\mathrm{FH}}$-cells was originally thought to reflect their B helper capabilities [90], but a recent report showed that it actually inhibits B-cell responses in mice in vivo [137]. We obtained very similar results with both tonsillar $\mathrm{T}_{\mathrm{FH}}$-cells and with circulating $\mathrm{T}_{\mathrm{FH}}$-like subsets in humans [45]. Therefore, IL-10 production by $\mathrm{T}_{\mathrm{FH}}$-cells, similar to IL-10 produced by regulatory T-cells, might reflect disease activity, but is not necessarily playing a pathogenic role (Fig. 2).

\subsection{IL-10 producing extra-follicular helper T-cell subsets promote short- lived autoantibody responses}

Although $\mathrm{T}_{\mathrm{FH}}$ are the most potent $\mathrm{B}$ helper T-cells, also other $\mathrm{CD} 4{ }^{+} \mathrm{T}$ cell populations can promote B-cell responses [83,87,138]. In particular, extra-follicular B helper T-cells have been identified in human tonsils [139], and are drivers of autoantibody production in the MRL/ lpr lupus mouse model [140]. However, the involvement of $\mathrm{T}_{\mathrm{FH}}$ versus extra-follicular B helper T-cells varies strongly in different mouse models [129]. In the NZB/W model, where IL-10 plays a pathogenic role, both long-lived and short-lived plasma blasts contributed to antidsDNA IgG production [141]. Since short-lived plasma blasts can be generated outside of germinal centres, this suggests a role for both $\mathrm{T}_{\mathrm{FH}}$ and extra-follicular B helper T-cells. In SLE patients, the levels of pathogenic anti-dsDNA autoantibodies are highly dynamic [142], and rapidly drop in some patients upon Rituximab treatment [7]. Since long-lived plasma cells are not targeted by Rituximab, these findings suggest that anti-dsDNA autoantibodies in these patients were produced predominantly by short-lived plasma blasts. Moreover, a fraction of IgG autoantibodies is not somatically hyper-mutated [5], further suggesting 


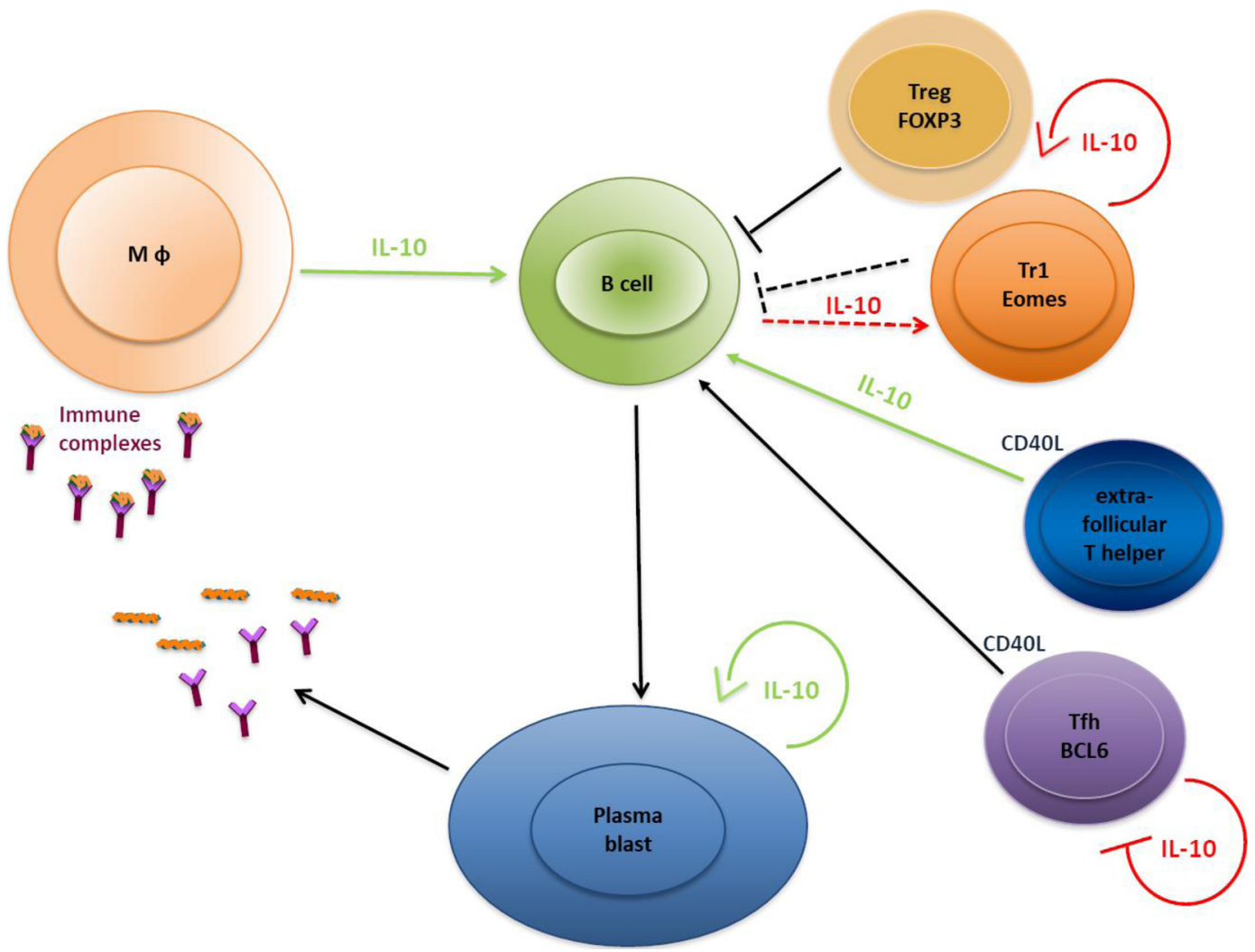

Fig. 2. Different roles of IL-10 produced by individual immune cell subsets in the regulation of B-cell responses and in SLE. IL-10 in SLE is produced by myeloid cells like macrophages (M $\Phi)$ as well as by different subsets of the B- and T-cell compartments. The current evidence suggests that IL-10 produced by macrophages and/or by extra-follicular helper T-cells promotes B-cells to differentiate to immunoglobulin-secreting plasma blasts, that might in addition promote their own generation by an autocrine IL-10 loop (green arrows). Conversely, recent evidence suggests that IL-10 produced by BCL6-expressing follicular helper T-cells $\left(\mathrm{T}_{\mathrm{FH}}\right)$ may represent a negative feed-back loop (red line). In addition, there is a regulatory loop between Il-10 producing regulatory B-cells and T-cells that appears to be disturbed in SLE (dotted red lines): IL-10 produced by regulatory B-cells could induce Tr1-cells, which similar to FOXP3 ${ }^{+}$Tregs lack CD40 L and inhibit B-cell responses, but are dysfunctional in SLE. IL-10 derived from regulatory T-cells does not directly inhibit B-cell responses, but could be important to maintain the suppressive functions of $\mathrm{FOXP}^{+}$and $\mathrm{FOXP}^{-}$regulatory T-cells in target tissues (red inhibitory arrow).

a contribution of extra-follicular B-cell responses to autoantibody production in SLE.

A recent report provided evidence for a role of extra-follicular B helper T-cells in human lupus nephritis [78]. Thus, $\mathrm{CD} 4{ }^{+} \mathrm{PD} 1{ }^{+} \mathrm{CXCR}^{+}{ }^{+} \mathrm{CXCR} 5{ }^{-} \mathrm{T}$-cells, i.e. activated Th1-cells [143] that produce IL-10 and IFN- $\gamma$, were expanded in the blood of SLE patients and were enriched in the kidney. They induced IgG in an IL-10- and succinate-dependent manner in vitro, consistent with a pathogenic role. However, if CXCR5 ${ }^{-} \mathrm{PD} 1^{+}$Th1-cells are also able to mediate B cell help in vivo was not investigated. IL-10 producing Th1-cells with similar characteristics could be induced by IFN- $\alpha$-producing pDC in vitro, which may possibly explain their abundance in SLE patients [78]. Notably, RNA sequencing revealed that these pDC-primed, IL-10 producing Th1cells also expressed CCR5, Eomes and Granzymes, suggesting that they are closely related to Eomes ${ }^{+} \operatorname{Tr} 1$-cells [108] (see Review by Hill and colleague in this special issue). Indeed, Eomes ${ }^{+} \mathrm{Tr} 1$-cells have a similar phenotype [93], and are consequently contained among $\mathrm{CD} 4{ }^{+} \mathrm{PD} 1{ }^{+} \mathrm{CXCR}^{+}{ }^{+} \mathrm{CXCR}^{-}{ }^{-}$-cells (unpublished observation). Notably, human extra-follicular B helper T-cells and circulating Th1-cells can be distinguished from Eomes ${ }^{+}$Tr1-cells by IL-7R expression $[79,139,143]$. Future research should thus clarify if IL-10 and IFN- $\gamma$ producing Th1-cells and Tr1-cells have different roles in B-cell responses and SLE.

We have obtained independent evidence for a pathogenic role of IL10 producing, extra-follicular B helper T-cells in SLE. Some time ago we identified a population of IL-10 producing, auto-reactive CCR6 $^{+}$ memory T-cells [120]. Notably, the "physiological" auto-reactivity in healthy individuals among helper T-cells is largely confined to
CCR6 $^{+}$T-cells, suggesting that they play a key role in autoimmune diseases [120]. Consistently, we and others provided evidence for a pathogenic role of auto-reactive $\mathrm{CCR} 6^{+} \mathrm{T}$-cells that produce pro-inflammatory cytokines in multiple sclerosis [144,145]. Autoreactive CCR6 $^{+}$T-cells have a peculiar context-dependent behaviour. They produce IL-10, but not other cytokines, upon interaction with DC presenting self-MHC. However, they express IL-2 and CD40 L in addition upon stimulation with recall antigens like tetanus toxoid. Since tetanus vaccination protects by the induction of neutralising antibodies, we speculated that $\mathrm{CCR}^{+}{ }^{+}$memory T-cells might provide help for B-cell in recall responses [120]. Indeed, we found recently that CCR6 ${ }^{+} \mathrm{IL}_{-}-\mathrm{R}^{+}$ from human tonsils and from spleens of IL-10 reporter mice induced IgG in a partially IL-10-dependent manner in vitro and in vivo [45]. IL-10 producing CCR $^{+}{ }^{+}$T-cells are central memory-like cells that lack BCL6, as well as IL-17 and IFN- $\gamma$ producing capacities, indicating that they are distinct from $\mathrm{T}_{\mathrm{FH}}$ as well as from Th1 and Th17-cells with B helper functions $[78,87,125]$. Importantly, they accumulate systemically in pristane-induced lupus-like disease [146], and selectively in SLE patients with anti-dsDNA antibodies. In SLE patients, but not in healthy individuals, they spontaneously induced IgG including anti-dsDNA antibodies in the absence of exogenous TCR agonists. Moreover, they were highly abundant in lymph nodes of SLE patients and interacted with Bcells, but were excluded from germinal centres. These data suggest that IL-10 producing CCR6 ${ }^{+}$T-cells are a distinct population of extra-follicular B-helper T-cells that are autoreactive and drive the generation of short-lived autoantibody-producing plasma blasts in SLE [45]

In summary, there is accumulating evidence that besides $\mathrm{T}_{\mathrm{FH}}$ also extra-follicular B helper T-cells contribute to the aberrant IL-10 and 
autoantibody production in SLE. This pathway appears to be particular important for the dynamic induction of pathogenic anti-DNA autoantibodies [142].

\section{Summary/Outlook}

It is increasingly recognised that Interleukin-10, the prototypic antiinflammatory cytokine, has a paradoxical pathogenic activity in the systemic autoimmune disease SLE. It seems likely that the capacity of IL-10 to stimulate autoantibody production by autoreactive B-cells is key to its pathogenic role, but other, yet unidentified, pathways may also contribute. The current evidence suggests that IL-10 is produced by multiple cellular sources in lupus, and that IL-10 produced by different immune cells might have different functions that depend on the context or the microenvironment. In particular, $\mathrm{CD} 4^{+} \mathrm{T}$-cells are likely to contribute importantly to the aberrant IL-10 production in lupus. IL-10 is produced by different subsets of regulatory and follicular, as well as extra-follicular B helper T-cells, which play different roles in SLE. Since follicular and extra-follicular B-cell responses are preferentially targeted by different therapeutic strategies [7,147], understanding their relative contributions in individual SLE patients may hold promise for personalised therapies with higher efficacies.

\section{Acknowledgment}

This work was supported by the Romeo ed Enrica Invernizzi Foundation and by the Gruppo italiano per la lotta contro il LES ONLUS.

\section{References}

[1] R. Gualtierotti, M. Biggioggero, A.E. Penatti, P.L. Meroni, Updating on the pathogenesis of systemic lupus erythematosus, Autoimmun. Rev. 10 (2010) 3-7.

[2] J. Geginat, et al., The light and the dark sides of Interleukin-10 in immunemediated diseases and cancer, Cytokine Growth Factor Rev. (2016).

[3] P.S. Ramos, et al., A comprehensive analysis of shared loci between systemic lupus erythematosus (SLE) and sixteen autoimmune diseases reveals limited genetic overlap, PLoS Genet. 7 (2011) e1002406.

[4] Y. Deng, B.P. Tsao, Genetic susceptibility to systemic lupus erythematosus in the genomic era, Nat. Rev. Rheumatol. 6 (2010) 683-692.

[5] B. Mietzner, et al., Autoreactive IgG memory antibodies in patients with systemic lupus erythematosus arise from nonreactive and polyreactive precursors, Proc. Natl. Acad. Sci. U.S.A. 105 (2008) 9727-9732.

[6] W. Stohl, et al., Belimumab reduces autoantibodies, normalizes low complement levels, and reduces select B cell populations in patients with systemic lupus erythematosus, Arthritis Rheum. 64 (2012) 2328-2337.

[7] R. Gualtierotti, et al., Successful sequential therapy with rituximab and belimumab in patients with active systemic lupus erythematosus: a case series, Clin. Exp. Rheumatol. 36 (2018) 643-647.

[8] R. Magro, Biological therapies and their clinical impact in the treatment of systemic lupus erythematosus, Ther. Adv. Musculoskelet. Dis. 11 (2019) $1759720 X 19874309$.

[9] Y. Ghodke-Puranik, T.B. Niewold, Immunogenetics of systemic lupus erythematosus: a comprehensive review, J. Autoimmun. 64 (2015) 125-136.

[10] D. Finke, M.L. Eloranta, L. Ronnblom, Endogenous type I interferon inducers in autoimmune diseases, Autoimmunity 42 (2009) 349-352.

[11] L. Ronnblom, Potential role of IFNalpha in adult lupus, Arthritis Res. Ther. 12 (Suppl 1) (2010) S3.

[12] N. Seredkina, J. Van Der Vlag, J. Berden, E. Mortensen, O.P. Rekvig, Lupus nephritis - enigmas, conflicting models and an emerging concept, Mol Med (2013).

[13] O.P. Rekvig, Systemic Lupus Erythematosus: Definitions, Contexts, Conflicts, Enigmas, Front. Immunol. 9 (2018) 387.

[14] Y. Rahbar Saadat, et al., The role of microbiota in the pathogenesis of lupus: Dose it impact lupus nephritis? Pharmacol. Res. 139 (2019) 191-198.

[15] A.L. Astier, D.A. Hafler, Abnormal Tr1 differentiation in multiple sclerosis, J. Neuroimmunol. 191 (2007) 70-78.

[16] W.F. Chen, A. Zlotnik, IL-10: a novel cytotoxic T cell differentiation factor, J. Immunol. 147 (1991) 528-534.

[17] N.F. Go, et al., Interleukin 10, a novel B cell stimulatory factor: unresponsiveness of X chromosome-linked immunodeficiency B cells, J. Exp. Med. 172 (1990) $1625-1631$.

[18] Y. Levy, J.C. Brouet, Interleukin-10 prevents spontaneous death of germinal center B cells by induction of the bcl-2 protein, J. Clin. Invest. 93 (1994) 424-428.

[19] R. Kuhn, J. Lohler, D. Rennick, K. Rajewsky, W. Muller, Interleukin-10-deficient mice develop chronic enterocolitis, Cell 75 (1993) 263-274.

[20] J.L. Fornek, et al., Critical role for Stat3 in T-dependent terminal differentiation of
IgG B cells, Blood 107 (2006) 1085-1091.

[21] S.O. Yoon, X. Zhang, P. Berner, Y.S. Choi, IL-21 and IL-10 have redundant roles but differential capacities at different stages of Plasma Cell generation from human Germinal Center B cells, J. Leukoc. Biol. 86 (2009) 1311-1318.

[22] R. Ettinger, et al., IL-21 induces differentiation of human naive and memory B cells into antibody-secreting plasma cells, J. Immunol. 175 (2005) 7867-7879.

[23] H. Ishida, R. Hastings, J. Kearney, M. Howard, Continuous anti-interleukin 10 antibody administration depletes mice of Ly-1 B cells but not conventional B cells, J. Exp. Med. 175 (1992) 1213-1220.

[24] P. Youinou, Y. Renaudineau, The paradox of CD5-expressing B cells in systemic lupus erythematosus, Autoimmun. Rev. 7 (2007) 149-154.

[25] H. Ishida, et al., Continuous administration of anti-interleukin 10 antibodies delays onset of autoimmunity in NZB/W F1 mice, J. Exp. Med. 179 (1994) 305-310.

[26] E.L. Williams, S. Gadola, C.J. Edwards, Anti-TNF-induced lupus, Rheumatology Oxford (Oxford) 48 (2009) 716-720.

[27] L. Ban, et al., Selective death of autoreactive T cells in human diabetes by TNF or TNF receptor 2 agonism, Proc Natl Acad Sci U S A 105 (2008) 13644-13649.

[28] H. Peng, et al., Role of interleukin-10 and interleukin-10 receptor in systemic upus erythematosus, Clin. Rheumatol. (2013).

[29] Z. Yin, et al., IL-10 regulates murine lupus, J. Immunol. 169 (2002) 2148-2155.

[30] K. Bride, D. Teachey, Autoimmune lymphoproliferative syndrome: more than a FAScinating disease, F1000Res 6 (1928) (2017).

[31] Y.B. Park, et al., Elevated interleukin-10 levels correlated with disease activity in systemic lupus erythematosus, Clin. Exp. Rheumatol. 16 (1998) 283-288.

[32] M. Uchida, et al., Anti-IL-10 antibody in systemic lupus erythematosus, Open Access Rheumatol. 11 (2019) 61-65.

[33] P. Lopez, C. Gutierrez, A. Suarez, IL-10 and TNFalpha genotypes in SLE, J. Biomed. Biotechnol. 2010 (2010) 838390.

[34] A.W. Gibson, et al., Novel single nucleotide polymorphisms in the distal IL-10 promoter affect IL-10 production and enhance the risk of systemic lupus erythematosus, J. Immunol. 166 (2001) 3915-3922.

[35] D. Sakurai, et al., Preferential binding to Elk-1 by SLE-associated IL10 risk allele upregulates IL10 expression, PLoS Genet. 9 (2013) e1003870.

[36] M. Zhao, et al., Hypomethylation of IL10 and IL13 promoters in CD4 + T cells of patients with systemic lupus erythematosus, J. Biomed. Biotechnol. 2010 (2010) 931018.

[37] L. Llorente, et al., Spontaneous production of interleukin-10 by B lymphocytes and monocytes in systemic lupus erythematosus, Eur. Cytokine Netw. 4 (1993) 421-427.

[38] E. Hagiwara, M.F. Gourley, S. Lee, D.K. Klinman, Disease severity in patients with systemic lupus erythematosus correlates with an increased ratio of interleukin10:interferon-gamma-secreting cells in the peripheral blood, Arthritis Rheum. 39 (1996) 379-385.

[39] L. Llorente, et al., Role of interleukin 10 in the B lymphocyte hyperactivity and autoantibody production of human systemic lupus erythematosus, J. Exp. Med. 181 (1995) 839-844.

[40] C.T. Ravirajan, et al., Effect of neutralizing antibodies to IL-10 and C5 on the rena damage caused by a pathogenic human anti-dsDNA antibody, Rheumatology Oxford (Oxford) 43 (2004) 442-447.

[41] L. Llorente, et al., Clinical and biologic effects of anti-interleukin-10 monoclonal antibody administration in systemic lupus erythematosus, Arthritis Rheum. 43 (2000) 1790-1800.

[42] E. Zigmond, et al., Macrophage-restricted interleukin-10 receptor deficiency, but not IL-10 deficiency, causes severe spontaneous colitis, Immunity 40 (2014) 720-733.

[43] S. Huber, et al., Th17 cells express interleukin-10 receptor and are controlled by Foxp3 and Foxp3 + regulatory CD4 + T cells in an interleukin-10-dependent manner, Immunity 34 (2011) 554-565.

[44] F. Rousset, et al., Interleukin 10 is a potent growth and differentiation factor for activated human B lymphocytes, Proc. Natl. Acad. Sci. U.S.A. 89 (1992) 1890-1893.

[45] F. Facciotti, et al., Evidence for a Pathogenic Role of Extra-follicular, IL-10 Producing CCR6 + B-helper T-cells in Systemic Lupus Erythematosus, submitted (2019).

[46] H. Groux, M. Bigler, J.E. de Vries, M.G. Roncarolo, Inhibitory and stimulatory effects of IL-10 on human CD8 + T cells, J. Immunol. 160 (1998) 3188-3193.

[47] J. Emmerich, et al., IL-10 directly activates and expands tumor-resident CD8(+) T cells without de novo infiltration from secondary lymphoid organs, Cancer Res. 72 (2012) 3570-3581.

[48] G. Nizzoli, et al., IL-10 promotes homeostatic proliferation of human CD8(+) memory T cells and, when produced by CD1c $(+)$ DCs, shapes naive CD8 $(+)$ T-cell priming, Eur. J. Immunol. 46 (2016) 1622-1632.

[49] D.M. Gravano, K.K. Hoyer, Promotion and prevention of autoimmune disease by CD8 + T cells, J. Autoimmun. 45 (2013) 68-79.

[50] D. Shah, R. Kiran, A. Wanchu, A. Bhatnagar, Soluble granzyme B and cytotoxic T lymphocyte activity in the pathogenesis of systemic lupus erythematosus, Cell. Immunol. 269 (2011) 16-21.

[51] R. Baek Sorensen, et al., Melanoma inhibitor of apoptosis protein (ML-IAP) specific cytotoxic T lymphocytes cross-react with an epitope from the auto-antigen SS56, J. Invest. Dermatol. 129 (2009) 1992-1999.

[52] R. Sabat, et al., Biology of interleukin-10, Cytokine Growth Factor Rev. 21 (2010) 331-344.

[53] J.C. Crispin, M.I. Vargas-Rojas, A. Monsivais-Urenda, J. Alcocer-Varela, Phenotype and function of dendritic cells of patients with systemic lupus erythematosus, Clin. Immunol. 143 (2012) 45-50.

[54] Z. Sun, et al., Serum IL-10 from systemic lupus erythematosus patients suppresses 
the differentiation and function of monocyte-derived dendritic cells, J. Biomed. Res. 26 (2012) 456-466.

[55] P. Ancuta, et al., Opposite effects of IL-10 on the ability of dendritic cells and macrophages to replicate primary CXCR4-dependent HIV-1 strains, J. Immunol. 166 (2001) 4244-4253.

[56] S.E. Macatonia, T.M. Doherty, S.C. Knight, A. O'Garra, Differential effect of IL-10 on dendritic cell-induced T cell proliferation and IFN-gamma production, J. Immunol. 150 (1993) 3755-3765.

[57] M. Tokuda, S. Nagaoka, M. Torii, Interleukin-10 receptor expression in human dental pulp cells in response to lipopolysaccharide from Prevotella intermedia, $J$ Endod 29 (2003) 48-50.

[58] P.A. Becherel, et al., Interleukin-10 inhibits IgE-mediated nitric oxide synthase induction and cytokine synthesis in normal human keratinocytes, Eur. J. Immunol. 25 (1995) 2992-2995 Issn: 0014-2980.

[59] M. Seifert, et al., Keratinocyte unresponsiveness towards interleukin-10: lack of specific binding due to deficient IL-10 receptor 1 expression, Exp. Dermatol. 12 (2003) 137-144.

[60] J.M. Lanis, et al., Tryptophan metabolite activation of the aryl hydrocarbon receptor regulates IL-10 receptor expression on intestinal epithelia, Mucosal Immunol. 10 (2017) 1133-1144.

[61] N.J. Davidson, et al., T helper cell 1-type CD4 + T cells, but not B cells, mediate colitis in interleukin 10-deficient mice, J. Exp. Med. 184 (1996) 241-251.

[62] L.E. Rees, et al., The interleukin-10-1082 G/A polymorphism: allele frequency in different populations and functional significance, Cell. Mol. Life Sci. 59 (2002) 560-569.

[63] L. Larsson, L. Rymo, T. Berglundh, Sp1 binds to the G allele of the-1087 polymorphism in the IL-10 promoter and promotes IL-10 mRNA transcription and protein production, Genes Immun. 11 (2010) 181-187.

[64] J. Martinez, et al., Noncanonical autophagy inhibits the autoinflammatory, lupuslike response to dying cells, Nature 533 (2016) 115-119.

[65] E.Y. Chung, J. Liu, Y. Zhang, X. Ma, Differential expression in lupus-associated IL10 promoter single-nucleotide polymorphisms is mediated by poly(ADP-ribose) polymerase-1, Genes Immun. 8 (2007) 577-589.

[66] A.J. Smith, S.E. Humphries, Cytokine and cytokine receptor gene polymorphisms and their functionality, Cytokine Growth Factor Rev. 20 (2009) 43-59.

[67] B. Haringer, L. Lozza, B. Steckel, J. Geginat, Identification and characterization of IL-10/IFN-gamma-producing effector-like T cells with regulatory function in human blood, J. Exp. Med. 206 (2009) 1009-1017.

[68] A. Csiszar, G. Nagy, P. Gergely, T. Pozsonyi, E. Pocsik, Increased interferon-gamma (IFN-gamma), IL-10 and decreased IL-4 mRNA expression in peripheral blood mononuclear cells (PBMC) from patients with systemic lupus erythematosus (SLE), Clin. Exp. Immunol. 122 (2000) 464-470.

[69] S.J. DiMartino, W. Yuan, P. Redecha, L.B. Ivashkiv, J.E. Salmon, Insoluble immune complexes are most effective at triggering IL-10 production in human monocytes and synergize with TLR ligands and C5a, Clin. Immunol. 127 (2008) 56-65.

[70] J. Ronnelid, A. Tejde, L. Mathsson, K. Nilsson-Ekdahl, B. Nilsson, Immune complexes from SLE sera induce IL10 production from normal peripheral blood mononuclear cells by an FcgammaRII dependent mechanism: implications for a possible vicious cycle maintaining B cell hyperactivity in SLE, Ann. Rheum. Dis. 62 (2003) 37-42.

[71] H. Joo, et al., Serum from patients with SLE instructs monocytes to promote IgG and IgA plasmablast differentiation, J. Exp. Med. 209 (2012) 1335-1348.

[72] M. Manni, et al., IRF4-dependent and IRF4-Independent pathways contribute to DC dysfunction in lupus, PLoS One 10 (2015) e0141927.

[73] G. Heine, et al., Autocrine IL-10 promotes human B-cell differentiation into IgMor IgG-secreting plasmablasts, Eur. J. Immunol. 44 (2014) 1615-1621.

[74] N.A. Carter, et al., Mice lacking endogenous IL-10-producing regulatory B cells develop exacerbated disease and present with an increased frequency of Th1/Th17 but a decrease in regulatory T cells, J. Immunol. 186 (2011) 5569-5579.

[75] P.A. Blair, et al., CD19(+)CD24(hi)CD38(hi) B cells exhibit regulatory capacity in healthy individuals but are functionally impaired in systemic Lupus Erythematosus patients, Immunity 32 (2010) 129-140.

[76] M. Menon, P.A. Blair, D.A. Isenberg, C. Mauri, A regulatory feedback between plasmacytoid dendritic cells and regulatory B cells is aberrant in systemic lupus erythematosus, Immunity 44 (2016) 683-697.

[77] A. Fuchs, J.P. Atkinson, V. Fremeaux-Bacchi, C. Kemper, CD46-induced human Treg enhance B-cell responses, Eur. J. Immunol. 39 (2009) 3097-3109.

[78] S. Caielli, et al., A CD4(+) T cell population expanded in lupus blood provides B cell help through interleukin-10 and succinate, Nat. Med. 25 (2019) 75-81.

[79] F. Facciotti, et al., IL-10-producing forkhead box protein 3-negative regulatory $\mathrm{T}$ cells inhibit B-cell responses and are involved in systemic lupus erythematosus, J. Allergy Clin. Immunol. 137 (318-321) (2016) e315.

[80] T. Okamura, et al., TGF-beta3-expressing CD4 + CD25(-)LAG3 + regulatory T cells control humoral immune responses, Nat. Commun. 6 (2015) 6329.

[81] F. Meiler, S. Klunker, M. Zimmermann, C.A. Akdis, M. Akdis, Distinct regulation of IgE, IgG4 and IgA by T regulatory cells and toll-like receptors, Allergy 63 (2008) 1455-1463.

[82] L. Brockmann, et al., Molecular and functional heterogeneity of IL-10-producing CD4(+) T cells, Nat. Commun. 9 (2018) 5457.

[83] C.R. Ruprecht, A. Lanzavecchia, Toll-like receptor stimulation as a third signal required for activation of human naive B cells, Eur. J. Immunol. 36 (2006) 810-816.

[84] P. Schaerli, et al., CXC chemokine receptor 5 expression defines follicular homing T cells with B cell helper function, J. Exp. Med. 192 (2000) 1553-1562.

[85] D. Breitfeld, et al., Follicular B helper T cells express CXC chemokine receptor 5, localize to B cell follicles, and support immunoglobulin production, J. Exp. Med.
192 (2000) 1545-1552.

[86] R. Morita, et al., Human blood CXCR5 $(+) \mathrm{CD} 4(+) \mathrm{T}$ cells are counterparts of $\mathrm{T}$ follicular cells and contain specific subsets that differentially support antibody secretion, Immunity 34 (2011) 108-121.

[87] F. Annunziato, et al., Phenotypic and functional features of human Th17 cells, J. Exp. Med. 204 (2007) 1849-1861.

[88] G. Del Prete, et al., Human IL-10 is produced by both type 1 helper (Th1) and type 2 helper (Th2) T cell clones and inhibits their antigen-specific proliferation and cytokine production, J. Immunol. 150 (1993) 353-360.

[89] C.E. Zielinski, et al., Pathogen-induced human TH17 cells produce IFN-gamma or IL-10 and are regulated by IL-1beta, Nature 484 (2012) 514-518.

[90] C.H. Kim, et al., Subspecialization of CXCR5 + T cells: B helper activity is focused in a germinal center-localized subset of CXCR5 + T cells, J. Exp. Med. 193 (2001) 1373-1381.

[91] S. Shivakumar, G.C. Tsokos, S.K. Datta, T cell receptor alpha/beta expressing double-negative (CD4-/CD8-) and CD4 + T helper cells in humans augment the production of pathogenic anti-DNA autoantibodies associated with lupus nephritis, J. Immunol. 143 (1989) 103-112.

[92] S. Sakaguchi, Naturally arising Foxp3-expressing CD25 + CD4 + regulatory T cells in immunological tolerance to self and non-self, Nat. Immunol. 6 (2005) 345-352.

[93] J.S. Alfen, et al., Intestinal IFN-gamma-producing Tr1-cells co-express CCR5 and PD-1, and down-regulate IL-10 in the inflamed gut of IBD patients, J. Allergy Clin. Immunol. (2018).

[94] C.L. Maynard, et al., Regulatory T cells expressing interleukin 10 develop from Foxp3 + and Foxp3- precursor cells in the absence of interleukin 10, Nat. Immunol. 8 (2007) 931-941.

[95] Y.P. Rubtsov, et al., Regulatory T cell-derived interleukin-10 limits inflammation at environmental interfaces, Immunity 28 (2008) 546-558.

[96] H.H. Uhlig, et al., Characterization of Foxp3 + CD4 + CD25 + and IL-10-secreting CD4 + CD25 + T cells during cure of colitis, J. Immunol. 177 (2006) 5852-5860.

[97] H.D. Ochs, E. Gambineri, T.R. Torgerson, IPEX, FOXP3 and regulatory T-cells: a model for autoimmunity, Immunol. Res. 38 (2007) 112-121.

[98] M.W. Appleby, F. Ramsdell, Scurfy, the Foxp3 locus, and the molecular basis of peripheral tolerance, Curr. Top. Microbiol. Immunol. 321 (2008) 151-168.

[99] M.A. Linterman, et al., Foxp3 + follicular regulatory T cells control the germinal center response, Nat. Med. 17 (2011) 975-982.

[100] K. Ohl, K. Tenbrock, Regulatory T cells in systemic lupus erythematosus, Eur. J. Immunol. 45 (2015) 344-355.

[101] M. Paroni, et al., Uncontrolled IL-17 production by intraepithelial lymphocytes in a case of non-IPEX autoimmune enteropathy, Clin. Transl. Gastroenterol. 7 (2016) e182.

[102] N. Moes, et al., Reduced expression of FOXP3 and regulatory T-cell function in severe forms of early-onset autoimmune enteropathy, Gastroenterology 139 (2010) 770-778.

[103] H. Groux, et al., A CD4 + T-cell subset inhibits antigen-specific T-cell responses and prevents colitis, Nature 389 (1997) 737-742.

[104] M.G. Roncarolo, S. Gregori, R. Bacchetta, M. Battaglia, N. Gagliani, The biology of t regulatory type 1 cells and their therapeutic application in immune-mediated diseases, Immunity 49 (2018) 1004-1019.

[105] H. Le Buanec, et al., IFN-alpha and CD46 stimulation are associated with active lupus and skew natural $\mathrm{T}$ regulatory cell differentiation to type 1 regulatory $\mathrm{T}$ (Tr1) cells, Proc. Natl. Acad. Sci. U.S.A. 108 (2011) 18995-19000.

[106] S. Sumitomo, et al., Identification of tonsillar CD4(+)CD25(-)LAG3(+) T cells as naturally occurring IL-10-producing regulatory $\mathrm{T}$ cells in human lymphoid tissue, J. Autoimmun. 76 (2017) 75-84.

[107] P. Zhang, et al., Eomesodermin promotes the development of type 1 regulatory T (TR1) cells, Sci. Immunol. 2 (2017).

[108] P. Gruarin, et al., Eomesodermin controls a unique differentiation program in human IL-10 and IFN-gamma coproducing regulatory T cells, Eur. J. Immunol. 49 (2019) 96-111.

[109] A. Schoenbrunn, et al., A converse 4-1BB and CD40 ligand expression pattern delineates activated regulatory $\mathrm{T}$ cells (Treg) and conventional $\mathrm{T}$ cells enabling direct isolation of alloantigen-reactive natural Foxp3 + Treg, J. Immunol. 189 (2012) 5985-5994.

[110] D. Gray, K. Siepmann, G. Wohlleben, CD40 ligation in B cell activation, isotype switching and memory development, Semin. Immunol. 6 (1994) 303-310.

[111] T. Kawabe, et al., The immune responses in CD40-deficient mice: impaired immunoglobulin class switching and germinal center formation, Immunity 1 (1994) $167-178$.

[112] K. Ozaki, et al., A critical role for IL-21 in regulating immunoglobulin production, Science 298 (2002) 1630-1634.

[113] Y. Iwasaki, et al., Egr-2 transcription factor is required for Blimp-1-mediated IL-10 production in IL-27-stimulated CD4 + T cells, Eur. J. Immunol. 43 (2013) 1063-1073.

[114] T. Okamura, et al., CD4 + CD25-LAG3 + regulatory T cells controlled by the transcription factor Egr-2, Proc. Natl. Acad. Sci. U.S.A. 106 (2009) 13974-13979.

[115] K. Morita, et al., Egr2 and Egr3 in regulatory T cells cooperatively control systemic autoimmunity through Ltbp3-mediated TGF-beta3 production, Proc. Natl. Acad. Sci. U. S. A. 113 (2016) E8131-E8140.

[116] N. Sugiyama, et al., Amelioration of human lupus-like phenotypes in MRL/lpr mice by overexpression of interleukin 27 receptor alpha (WSX-1), Ann. Rheum. Dis. 67 (2008) 1461-1467.

[117] M. Batten, et al., IL-27 supports germinal center function by enhancing IL-21 production and the function of T follicular helper cells, J. Exp. Med. 207 (2010) 2895-2906.

[118] R.R. Meka, S.H. Venkatesha, S. Dudics, B. Acharya, K.D. Moudgil, IL-27-induced 
modulation of autoimmunity and its therapeutic potential, Autoimmun. Rev. 14 (2015) 1131-1141.

[119] N.A. Carter, E.C. Rosser, C. Mauri, Interleukin-10 produced by B cells is crucial for the suppression of Th17/Th1 responses, induction of T regulatory type 1 cells and reduction of collagen-induced arthritis, Arthritis Res. Ther. 14 (2012) R32.

[120] L. Rivino, et al., CCR6 is expressed on an IL-10-producing, autoreactive memory T cell population with context-dependent regulatory function, J. Exp. Med. 207 (2010) 565-577.

[121] D.S. Shouval, et al., Interleukin-10 receptor signaling in innate immune cells regulates mucosal immune tolerance and anti-inflammatory macrophage function, Immunity 40 (2014) 706-719.

[122] S. Corinti, C. Albanesi, A. la Sala, S. Pastore, G. Girolomoni, Regulatory activity of autocrine IL-10 on dendritic cell functions, J. Immunol. 166 (2001) 4312-4318.

[123] K.W. Moore, A. O'Garra, R. de Waal Malefyt, P. Vieira, T.R. Mosmann, Interleukin10, Annu. Rev. Immunol. 11 (1993) 165-190.

[124] M. Akahoshi, et al., Th1/Th2 balance of peripheral T helper cells in systemic lupus erythematosus, Arthritis Rheum. 42 (1999) 1644-1648.

[125] S. Crotty, Follicular helper CD4 t cells (TFH), Annu. Rev. Immunol. 29 (2011) 621-663.

[126] B. Johansson-Lindbom, S. Ingvarsson, C.A. Borrebaeck, Germinal centers regulate human Th2 development, J. Immunol. 171 (2003) 1657-1666.

[127] A.G. Zaretsky, et al., T follicular helper cells differentiate from Th2 cells in response to helminth antigens, J. Exp. Med. 206 (2009) 991-999.

[128] P. Blanco, H. Ueno, N. Schmitt, T follicular helper (Tfh) cells in lupus: activation and involvement in SLE pathogenesis, Eur. J. Immunol. (2015).

[129] M.A. Linterman, et al., Follicular helper T cells are required for systemic autoimmunity, J. Exp. Med. 206 (2009) 561-576.

[130] M. Locci, et al., Human circulating PD-1CXCR3CXCR5 memory tfh cells are highly functional and correlate with broadly neutralizing HIV antibody responses, Immunity (2013).

[131] L. Rivino, et al., Chemokine receptor expression identifies Pre-T helper (Th)1, PreTh2, and nonpolarized cells among human CD4 + central memory T cells, J. Exp. Med. 200 (2004) 725-735.

[132] J. He, et al., Circulating precursor CCR7(lo)PD-1(hi) CXCR5(+) CD4(+) T cells indicate $\mathrm{Tfh}$ cell activity and promote antibody responses upon antigen reexposure, Immunity 39 (2013) 770-781.

[133] N. Simpson, et al., Expansion of circulating T cells resembling follicular helper T cells is a fixed phenotype that identifies a subset of severe systemic lupus erythematosus, Arthritis Rheum. 62 (2010) 234-244.

[134] C. Le Coz, et al., Circulating TFH subset distribution is strongly affected in lupus patients with an active disease, PLoS One 8 (2013) e75319.

[135] J.Y. Choi, et al., Circulating follicular helper-like T cells in systemic lupus erythematosus: association with disease activity, Arthritis Rheumatol 67 (2015) 988-999.

[136] C.E. Faliti, et al., P2X7 receptor restrains pathogenic Tfh cell generation in systemic lupus erythematosus, J. Exp. Med. 216 (2019) 317-336.

[137] P.F. Canete, et al., Regulatory roles of IL-10-producing human follicular T cells, J. Exp. Med. (2019).

[138] M. Mitsdoerffer, et al., Proinflammatory T helper type 17 cells are effective B-cell helpers, Proc. Natl. Acad. Sci. U.S.A. 107 (2010) 14292-14297.

[139] S.E. Bentebibel, N. Schmitt, J. Banchereau, H. Ueno, Human tonsil B-cell lymphoma 6 (BCL6)-expressing CD4 + T-cell subset specialized for B-cell help outside germinal centers, Proc Natl Acad Sci U S A 108 (2011) E488-497.

[140] J.M. Odegard, et al., ICOS-dependent extrafollicular helper T cells elicit IgG production via IL-21 in systemic autoimmunity, J. Exp. Med. 205 (2008) 2873-2886.

[141] B.F. Hoyer, et al., Short-lived plasmablasts and long-lived plasma cells contribute to chronic humoral autoimmunity in NZB/W mice, J. Exp. Med. 199 (2004) 1577-1584.

[142] D.S. Pisetsky, Anti-DNA antibodies-quintessential biomarkers of SLE, Nat. Rev. Rheumatol. 12 (2016) 102-110.

[143] J. Geginat, et al., The CD4-centered universe of human T cell subsets, Semin. Immunol. 25 (2013) 252-262.

[144] M. Paroni, et al., Recognition of viral and self-antigens by TH1 and TH1/TH17 central memory cells in patients with multiple sclerosis reveals distinct roles in immune surveillance and relapses, J. Allergy Clin. Immunol. (2017).

[145] Y. Cao, et al., Functional inflammatory profiles distinguish myelin-reactive T cells from patients with multiple sclerosis, Sci. Transl. Med. 7 (2015) 287ra274.

[146] H. Leiss, et al., Pristane-induced lupus as a model of human lupus arthritis: evolvement of autoantibodies, internal organ and joint inflammation, Lupus 22 (2013) 778-792

[147] T. Alexander, et al., Proteasome inhibition with bortezomib induces a therapeutically relevant depletion of plasma cells in SLE but does not target their precursors, Eur. J. Immunol. 48 (2018) 1573-1579. 